\title{
DO RELATIVISTIC ELECTRONS EITHER GAIN OR LOSE ENERGY, OUTSIDE OF EXTRAGALACTIC NUCLEI?
}

\author{
Lawrence Rudnick, Debora M. Katz-Stone, and Martha C. Anderson \\ Department of Astronomy, University of Minnesota, Minneapolis, MN 55455 \\ Received 1993 February 26; accepted 1993 May 25
}

\begin{abstract}
In the generally accepted models of high-luminosity extragalactic radio sources, the relativistic electron distribution evolves significantly outside of its source in the galactic nucleus; synchrotron losses occur throughout the source, and the harder (flatter) spectra seen in the hot spots are one indicator of relativistic particle acceleration. Our reanalysis and interpretation of the Carilli et al. (1991) data on Cygnus A, on the other hand, showed no evidence for evolution of the electron distribution function, and no evidence of the universally assumed injection power-law distribution. Having found a spectrum that describes an entire source, we have also introduced the technique of correcting synchrotron maps for frequency-dependent effects. In this way, a partial deconvolution of the underlying physical parameters is possible. In Cygnus A, this correction procedure led to the dominance of a hitherto unrecognized structure, an edge-brightened channel girdled by rings. We briefly discuss some implications of this work and our plans to continue this line of investigation.
\end{abstract}

Subject headings: acceleration of particles - galaxies: individual (Cygnus A) — galaxies: nuclei radiation mechanisms: nonthermal — radio continuum: galaxies

\section{INTRODUCTION}

The answer to the question posed in the title is, obviously, "yes"-relativistic electrons must both gain and lose energy outside of the nucleus of extragalactic radio sources. These energy transfers occur through the well-understood processes of adiabatic changes, synchrotron and inverse Compton radiation, as well as through a variety of possible stochastic and other particle acceleration mechanisms. The real question is the relative importance of each of these processes, and how they are linked to the dynamical and other features of the synchrotron source.

We focus our attention on the shape of the observed synchrotron spectrum, and how that shape varies throughout a source, as one important indicator of the underlying physical processes. Adiabatic changes, or changes in the scaling of the magnetic field, preserve the shape of the relativistic electron distribution, and hence the synchrotron spectrum. Processes in which electrons gain or lose an amount of energy not proportional to their energy will change the distribution and spectral shape. This important distinction between spectral shapepreserving and nonpreserving processes is almost always ignored in discussions of extragalactic radio sources.

We start with a very rough sketch of the conventional wisdom regarding the origin and evolution of the relativistic electrons in high-luminosity radio galaxies and QSOs. The relativistic plasma is assumed to be embedded in a much denser lower temperature plasma, which dominates the dynamics of the source. There are four main structural components in these sources, viz., the core, jets, hot spots, and lobes.

The electrons are initially produced in the galactic nucleus, which is usually observed as a compact, partially self-absorbed

\footnotetext{
${ }^{1}$ These data are from the Very Large Array, operated by the National Radio Astronomy Observatory, through Associated Universities, Inc.'s contract with the National Science Foundation.
}

core structure at centimeter wavelengths. These cores are well studied by VLBI techniques, and an optically thin, sometimes variable and sometimes superluminal parsec-scale jet is often found. The spectra of these jet components are generally poorly known, but appear consistent with a power law which is cut off at the low end by opacity (e.g., Marscher 1987).

On scales of $10^{0}-10^{1} \mathrm{kpc}$, jets (thin streams) of emission are often seen on one or both sides of the large-scale double source. The structure and overall energetics of the large-scale source are often used to argue that the jets are quite "efficient" (see discussions in Leahy 1991; Fraix-Burnet 1992) in transporting bulk kinetic energy to the hot spots and lobes without losing much of their energy through synchrotron or other losses. At least at centimeter wavelengths, there should thus be little change in the spectrum throughout the jet. However, declines in jet brightness are sometimes less than the level expected from adiabatic expansion when the jet broadens as it flows away from the core; this and observations of optical and X-ray jets (e.g., Biretta, Stern, \& Harris 1991) suggest that particle acceleration may be taking place throughout the jet.

At the terminal points of the source, compact high brightness features called "hot spots" are usually found. These have flatter radio spectra than the jets and lobes, often close to the strong (nonrelativistic) shock limit of $\alpha=-0.5$ for their spectral slope, (equivalent to a logarithmic slope of -2 in energy), where

$$
\alpha=\frac{d \log I(\nu)}{d \log \nu}
$$

The association of optical synchrotron radiation with the radio hot spots (Simkin 1978; Rudnick et al. 1981; Meisenheimer et al. 1989) argues quite strongly for in situ particle acceleration in a number of sources. 
After passing through the hot spots, the relativistic and nonrelativistic plasmas reverse their flow direction, and expand and flow back toward the nucleus, forming what are termed "lobes" or "bridges." The dominant changes to the electron population at this stage are adiabatic and synchrotron losses. The ubiquitous steepening of the spectrum in these lobes, as one goes from the hot spot back to the nucleus, is interpreted as "aging" due to synchrotron losses. This type of analysis is used to estimate flow speeds and ages for radio lobes.

If the above picture is correct, then we expect to see significant changes in the shape of the synchrotron spectra as one goes from the nucleus, through the jets, hot spots, and lobes. Contrary to all the standard interpretations, as described above, this is not what we found in our reanalysis of the data of Carilli et al. 1991 on Cygnus A, ${ }^{1}$ the prototypical radio galaxy (Katz-Stone, Rudnick, \& Anderson, 1993; see $\$ 2$ ).

As a byproduct of this work, we have also developed a method ( see $\S 3$ ) of partially deconvolving synchrotron images into their underlying physical parameters, once the shape of the spectrum is known. Applying this method to Cygnus A has led to some puzzles about physical conditions within the source (Katz-Stone \& Rudnick 1993). In $\S 4$, we describe some of our ideas about future investigations.

\section{GLOBAL SYNCHROTRON SPECTRUM}

\subsection{Spectrum in $\log I-\log \nu$ Space}

As described in Katz-Stone \& Rudnick (1993), we can write the observed intensity, $I\left(\nu_{\text {obs }}\right)$, at a fixed location and at observing frequency $\nu_{\text {obs }}$ as the integral of the emissivity, $\epsilon\left(\nu_{\text {obs }}\right)$, along the line of sight, where

$$
\epsilon\left(\nu_{\text {obs }}\right) \propto \frac{B^{0.5} \rho_{e}}{\gamma_{0}} \times \nu_{\text {obs }}^{0.5} f\left(\frac{\nu_{\text {obs }}^{0.5}}{B^{0.5} \gamma_{0}}\right)
$$

The function $f$ represents the electron energy distribution, $\rho_{e}$ is the relativistic electron number density, $B$ is the magnetic field strength, and $\gamma_{0}$ is an energy scaling factor. In general, each of these quantities is a function of position in the source. In this formulation, one can see that both the intensity and the spectral index at a fixed observing frequency $\nu_{\text {obs }}$ are complicated functions, e.g., of the magnetic field $B$. This is because changes in the magnetic field affect both the emissivity of an individual fixed-energy electron, as well as the energy of the electrons being observed. However, there is a simpler way to look at these changes, if observations are available over a broad frequency range. If one were observing two regions of plasma that were identical except for the strength of their $B$ fields, or of $\gamma_{0}$ (a simple energy scaling), then the spectra of the two regions would be related by a shift in both axes in $\log I-\log \nu$ space.

Figure $1 a$ shows the results of taking the Cygnus A data of Carilli et al. (1991) for several locations within the source, at up to nine frequencies, and shifting the set of points for each location in both $\log I$ and $\log \nu$, to find that they fit on a common spectral curve. The stars are data from one of the hot spots; the circles and triangles are from two different points in the lobes. Our conclusions from this plot, (and the two-color analysis described below) are as follows: (1) No power law is evident in Cygnus $A$, not at any place in the source and not at any frequency between $150 \mathrm{MHz}$ and $100 \mathrm{GHz}$. (2) A single

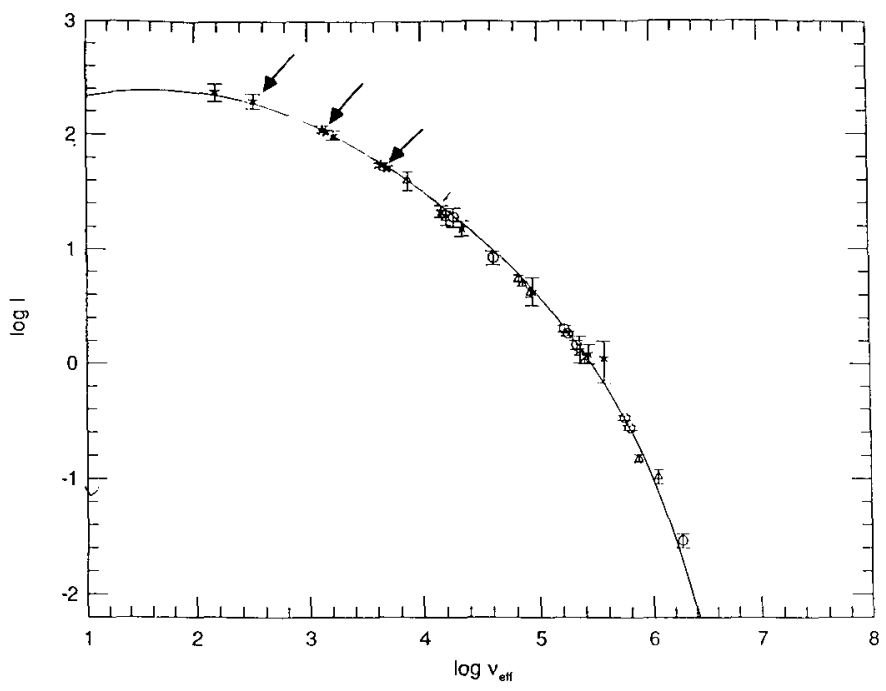

FIG. $1 a$

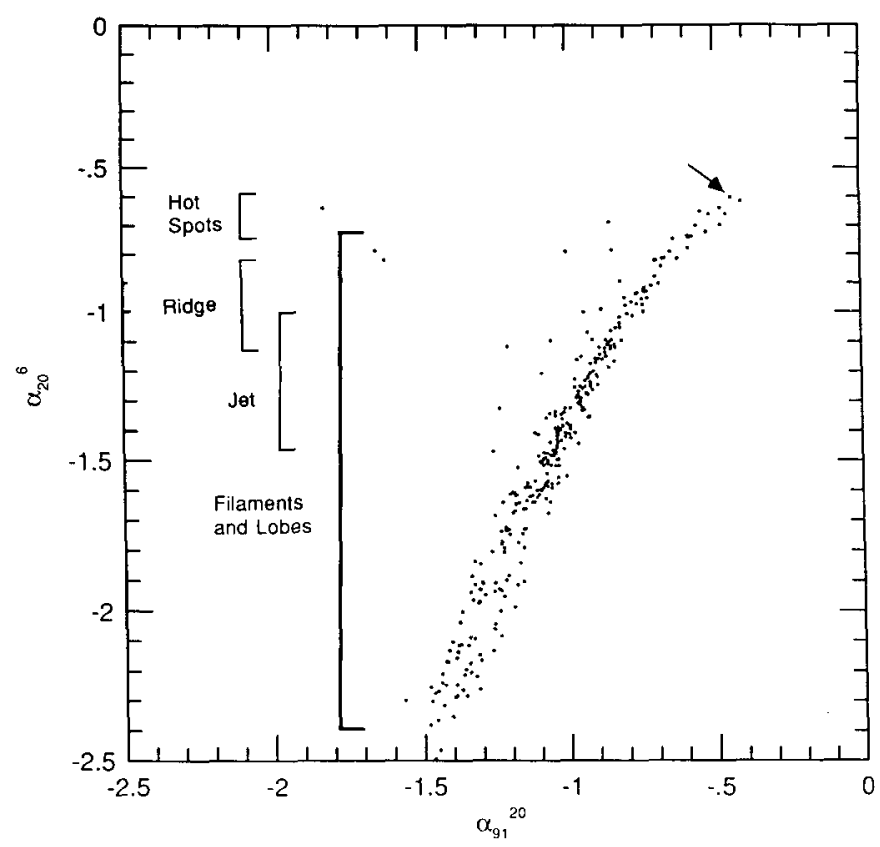

FIG. $1 b$

Fig. 1.- (a) The spectrum for three different locations in Cygnus A from the data of Carilli et al. 1991. The data for each location have been shifted in both $\log I$ and $\log \nu$ so as to fit on a common curve. The solid line is the spectral shape determined, not from these data, but from the three frequency data shown in $(b)$. For the data from the hot spot shown in $(a)$, we indicate these three frequencies with arrows. $(b)$ The color-color plot of Cygnus $A$, using data at $\lambda=91 \mathrm{~cm}, \lambda=20 \mathrm{~cm}$, and $\lambda=6 \mathrm{~cm}$. The arrow indicates the one hot spot point that appears in (a). Labels indicate the approximate regions spanned by various structural features in the source. The range indicated for the jet is quite uncertain, due to confusion from the background lobe ( see Katz-Stone et al. 1993).

spectral shape is consistent with the data on the jet, the hot spots, and the lobes.

There is thus, in Cygnus A, no evidence for either gains or losses in relativistic electron energies, other than simple (energy-independent) scalings. Many of the arguments in the literature for energy-dependent gain or loss processes are derived 
from measures of the spectral slope between two frequencies. This can be a very misleading process if one wants to understand the evolution of the relativistic electron distribution; changes in the underlying magnetic field with a curved electron distribution will themselves cause an apparent spectral change.

Another serious concern with the standard interpretations is that they are based on an initial power-law injection of relativistic electrons, while we find no evidence for this, at least in Cygnus A. If there were an initial power law, then the curvature at the low end of the spectrum, caused by low-energy cutoffs in the electron distribution, must fortuitously match up with the curvature at the high end, caused by synchrotron losses, to leave no power-law portion.

\subsection{Spectrum in Color-Color Space}

In practice, constructing the global spectrum by shifting of points is a quite difficult procedure without already having a good idea of the spectral shape. This is especially true for sources where data at only a few frequencies are available. In Katz-Stone et al. (1993) we introduced a new technique to determine the spectral shape using only data from three frequencies, and forming the color-color plot. This is how we actually determined the spectral shape for Cygnus $\mathrm{A}$, as plotted as a solid line in Figure $1 a$, and whose color-color plot is shown in Figure $1 b$.

In the "color-color" diagram, the spectral index between one pair of frequencies is plotted against the spectral index of another pair of frequencies, for all positions $(x, y)$ in the source. A point on the color-color diagram thus represents: $\left[\alpha_{i j}(x, y), \alpha_{j k}(x, y)\right]$, where $\alpha_{i j}(x, y)$ is the spectral index between frequencies $\nu_{i}$ and $\nu_{j}$ at the location $(x, y)$. The colorcolor diagram is a complementary tool to standard multifrequency analysis, and has some distinct advantages: (1) It is relatively easy to obtain, requiring observations at only three frequencies; (2) It displays information from all points in the source in a way that enables one to see the real connections between the various spectra; and (3) It is more sensitive to curvature in the spectrum than plots in $(\log I, \log \nu)$ space.

The color-color diagram can be used to see if there is more than one spectral shape present in the source. If the color-color points from all locations in a source follow a single curve, it is simplest to assume that there is indeed only one spectral shape in the source. The location along this curve is determined by the local value of $\frac{\nu_{\text {obs }}}{\gamma_{0}^{2} B}$. Thus, measuring the spectral index in the presence of a change in $\gamma_{0}^{2} B$ from location to location in a source is equivalent to observing at different frequencies, $\nu_{\text {obs }}$. In $(\log I, \log \nu)$ space, this can also be pictured as observing at a fixed $\nu_{\text {obs }}$, but being able to observe the whole spectral shape by having the spectrum shift in $\log \nu$ and $\log I$ as $\gamma_{0}^{2} B$ changes.

Using only information from this color-color diagram (at three observing frequencies covering 1 decade in frequency) we were thus able to generate the entire spectral shape $(\log I$, $\log \nu$ ) for Cygnus $\mathrm{A}$, as shown in Figure $1 a$, over an equivalent of 4 decades in frequency. We have indicated with arrows one set of corresponding points from the two different types of spectral plots. Color-color diagrams of two other radio sources are shown in Figure 2, and show similar shaped spectra to Cygnus A.

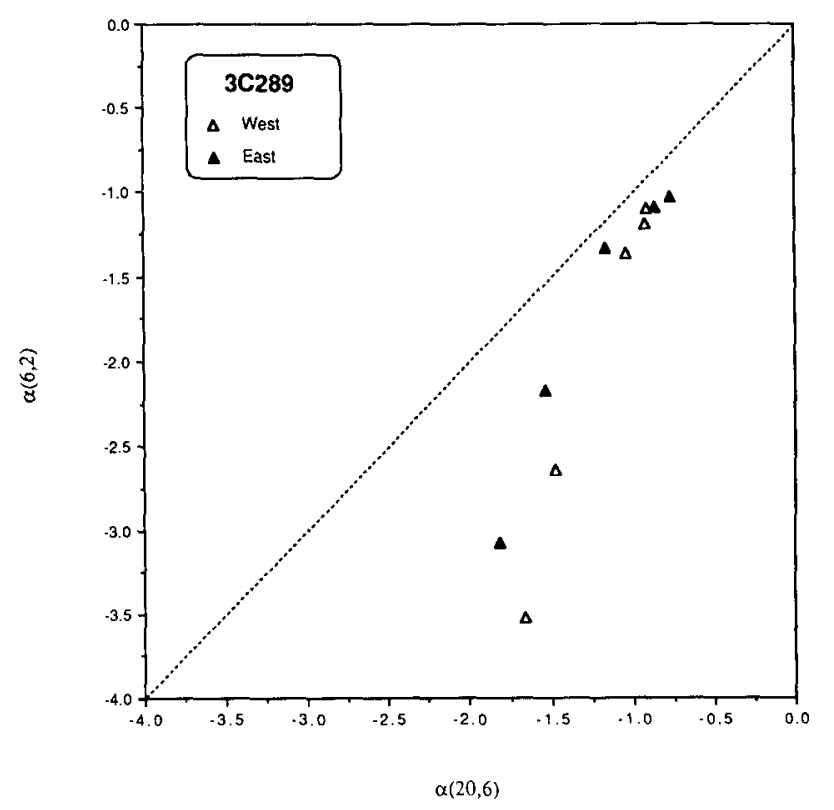

FiG. $2 a$

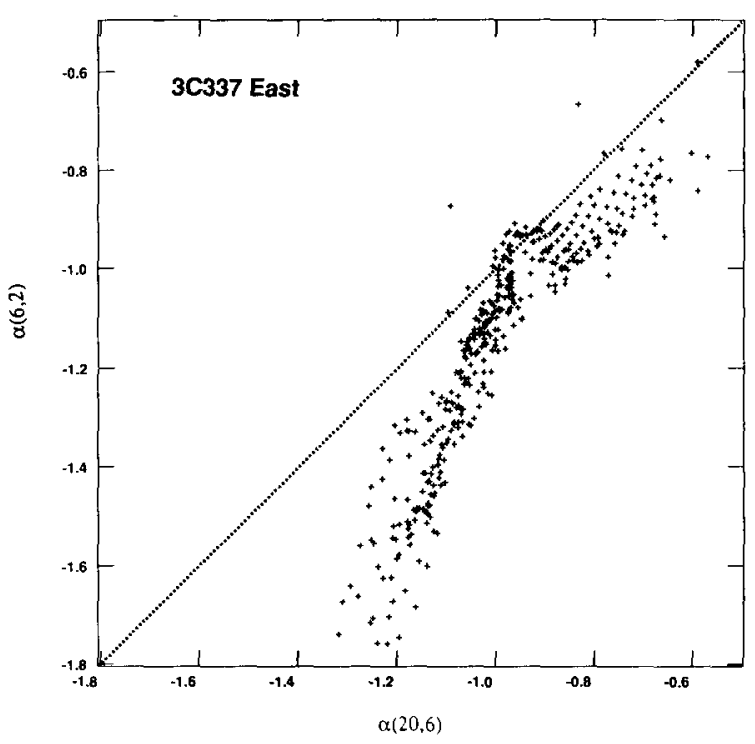

FiG. $2 b$

Fig. 2.-Color-color plots of two other extragalactic radio sources. $(a)$ 3C 289, from Liu, Pooley, \& Riley (1992) and $(b)$ 3C 337, from Pedelty et al. (1989). Note that the color-color plots all have different scales.

\section{TOWARD A PHYSICAL DECONVOLUTION OF TOTAL INTENSITY MAPS}

As seen in equation (2), the observed total intensity from a synchrotron source has a complicated functional dependence on the underlying physical parameters. This complication has usually been ignored because no tools have been developed to deal with frequency-dependent structures, or to isolate $\rho_{e}, \gamma_{0}$, and $B$, all of which contribute to the observed synchrotron brightness.

In the idealized case where a single power law of electrons is present throughout the source, the solutions for $\rho_{e}, \gamma_{0}$, and $B$ 

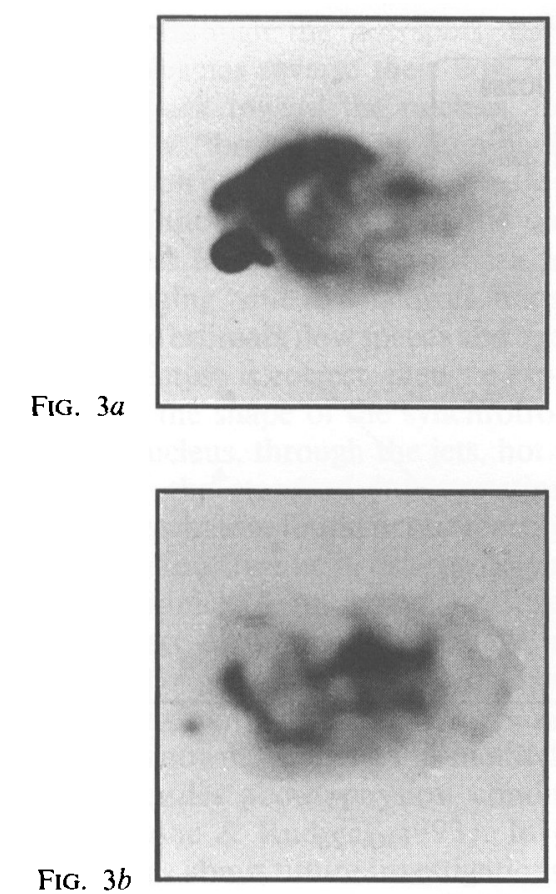

FIG. 3.-(a) The total intensity map at $\lambda=20 \mathrm{~cm}$ of the eastern lobe of Cygnus A, using the data of Carilli et al. 1991. (b) The frequency-independent, color-corrected map of the same region.

are completely degenerate; they cannot be isolated. However, when the electron distribution is curved, the appearance of the source does change as a function of observing frequency, and it is possible to partially isolate the contributions of the underlying physical parameters, i.e., to make maps which have a different functional dependence on the parameters than the original total intensity map (Katz-Stone \& Rudnick 1993). Figure $3 a$ shows the total intensity map of the eastern lobe of Cygnus A at $\lambda=20 \mathrm{~cm}$. Figure $3 b$ is a "color-corrected," or frequencyindependent version of the map, $I_{\text {corr }}$. As shown in Katz-Stone \& Rudnick (1993),

$$
I_{\text {corr }} \propto \rho_{e} B .
$$

This correction was performed by first determining the global synchrotron spectrum for Cygnus $A$, and then using the local spectral index between $\lambda=6 \mathrm{~cm}$ and $\lambda=20 \mathrm{~cm}$, to determine a spectral correction factor for each location.

Here we see a completely new view of Cygnus A, where the eastern lobe is dominated by a channel girdled by rings, and where the hot spots have almost disappeared. On the western side ( see Katz-Stone \& Rudnick 1993), the jet is not present in the $I_{\text {corr }}$ map. Maps of this new combination of parameters (eq. [3]) have not been seen before, and we do not know how to interpret them. Do the features dominating the $I_{\text {corr }}$ map have especially high values of $\rho_{e}$, or $B$, or both? Possible physical interpretations of this channel structure are discussed in KatzStone \& Rudnick (1993). It is not clear where this new information will lead, but we now have at our disposal a new tool for probing extragalactic radio sources.

\section{FUTURE WORK}

One important technical issue must be resolved, namely, how much of the shape we see in Cygnus A's spectrum directly reflects the underlying electron distribution, and how much is due to the distribution of magnetic fields along any line of sight. We are pursuing several ways of separating these two effects. We note that very broad magnetic field distributions will tend to make curved spectra more like power laws; this effect is important, e.g., in integrated spectra.

The discovery of a global synchrotron spectrum in Cygnus A casts doubts on models that invoke, e.g., spectral-shape-changing particle acceleration in hot spots, for which we have found no spectral evidence. Just as fundamentally, we see no evidence for the "aging" of the spectrum due to synchrotron losses. This does not rule out aging, but it means that this universally used assumption must be tested. In order to do this, we are studying "wide-angle-tail" radio sources, (e.g., O'Donoghue, Owen, \& Eilek, 1990), where we can follow the spectral shape through a region where it flattens downstream; we will look for evidence that either particle acceleration or synchrotron losses have modified the electron distribution along the way, or to see whether simple scaling changes in $B$ or in $\gamma_{0}$ could be responsible.

The low-frequency end of the spectrum is Cygnus A is especially problematic, since no power law is seen. It seems unlikely that a separate curvature-causing physical effect (e.g., a low electron-energy cutoff) would match smoothly on to the curvature already present in the higher frequency parts of the spectrum. In order to explore this low-energy regime, we are planning spectral shape observations of some high magnetic field "compact, steep-spectrum" sources.

We are also interested in seeing color-color diagrams for any sources where suitable data are available, and encourage others to construct them.

We are grateful to C. Carilli and R. A. Perley for providing the data on Cygnus A, and to them and T. W. Jones, J. P. Leahy, F. Owen, J. Eilek, and D. Clarke for challenging discussions. This work is supported, in part, through National Science Foundation grant AST-9100486 to the University of Minnesota.

\section{REFERENCES}

Biretta. J. A., Stern, C. P., \& Harris, D. E. 1991, AJ, 342, 128

Carilli, C., \& Perley, R. A. 1978, ApJ, 224, 39

Fraix-Burnet, D. 1992, A\&A, 259, 445

Katz-Stone, D., \& Rudnick, L. 1993, preprint

Katz-Stone, D. S., Rudnick, L., \& Anderson, M. C. 1993, ApJ, 407, 549

Leahy, J. P. 1991, in Beams and Jets in Astrophysics, ed. P. A. Hughes (Cambridge Univ. Press), 100

Liu, R., Pooley, G. G., \& Riley, J. M. 1992, MNRAS, 257, 545
Marscher, A. P. 1987, in Superluminal Radio Sources, ed. J. A. Zensus \& T. J. Pearson (Cambridge Univ. Press), 280

Meisenheimer, K., et al. 1989, A\&A, 219, 63

O'Donoghue, A., Owen, F. N., \& Eilek, J. A. 1990, ApJS, 72, 75

Pedelty, J. A., Rudnick, L., McCarthy, P. J., \& Spinrad, H. 1989, AJ, 98 , 1232

Rudnick, L., Saslaw, W. C., Crane, P., \& Tyson, J. A. 1981, ApJ, 246, 647

Simkin, S. 1978, ApJ, 234, 56 